\title{
Bilateral dentoalveolar asymmetries in female patients with adolescent idiopathic scoliosis
}

\author{
JOANNA LEWANDOWSKA ${ }^{1}$, JUSTYNA OPYDO-SZYMACZEK ${ }^{2 *}$, \\ KATARZYNA MEHR ${ }^{3}$, JAKUB GŁOWACKI ${ }^{4}$ \\ ${ }^{1}$ Postgraduate Studies in Scientific Research Methodology, Poznan University of Medical Sciences, Poznań, Poland. \\ ${ }^{2}$ Department of Pediatric Dentistry, Poznan University of Medical Sciences, Poznań, Poland. \\ ${ }^{3}$ Department of Dental Prosthetics, Pomeranian Medical University in Szczecin, Szczecin, Poland. \\ ${ }^{4}$ Department of General Orthopedics, Musculoskeletal Oncology and Trauma Surgery, \\ Poznan University of Medical Sciences, Poznań, Poland.
}

\begin{abstract}
Purpose: The aim of this cross-sectional research was to quantify left-right dentoalveolar fluctuating (FA) and directional asymmetries (DA) in patients with adolescent idiopathic scoliosis and to assess the relationship between these asymmetries and the side, location, and severity of scoliosis. Materials and methods: The study group comprised of 60 females aged 12-16 years, with idiopathic scoliosis, and 54 healthy controls. Measurements were made with the use of digital caliper on full-mouth dental casts. The following variables were measured from each subject: a shift of the incisor midline, left and right incisor overjet, deviation of canine and buccal segment relation as well as maxillary and mandibular arch chords. The data were statistically analyzed with significance taken as $p<0.05$. Results: Statistically significant differences in the mean shift of the incisor midline, buccal segment relation on the left and canine deviation on the left between the control group and the study group were detected ( $p=0.0419, p=0.0 .458$ and $p=0.0204$, respectively). FA of the midline deviation and canine deviation were statistically significantly higher for subjects with IS, compared to healthy controls ( $p=0.0315$ and $p=0.0415$, respectively). Neither direction of the curve nor apical vertebra's location or apical translation significantly affected the magnitude of dentoalveolar asymmetries. Conclusions: Our results confirmed that bilateral asymmetries are a common feature of the young females' occlusion. Individuals with IS show higher tendency to Angle Class II malocclusion, as well as higher FA of incisor midline discrepancy and canine deviation, compared to the controls.
\end{abstract}

Key words: dentoalveolar asymmetries, idiopathic scoliosis, fluctuating asymmetry, directional asymmetry

\section{Introduction}

Idiopathic scoliosis [IS] is a three-dimensional structural deformity of the spinal column, which appears before the end of the growth period, unassociated with other underlying pathologies such as malformations and neurological disorders. It is characterized by a sideto-side curvature of the spine of ten degrees or more, usually combined with a rotation of the vertebrae and a reduced kyphosis in thoracic curves [1].

Conventionally, idiopathic scoliosis is classified as infantile if it is detected before the child is three years old and juvenile if it is first noted in children from three to ten. When scoliosis appears in children between the age of ten and the time of skeletal maturation, it is called adolescent, a category that embraces $80 \%$ of the cases. Affecting girls more frequently than boys, the disorder is seen in about 1 to $4 \%$ of adolescents between the ages of 10 to 16 [1], [8], [24].

Changes in posture are a major consequence of idiopathic scoliosis. However, if the spinal curvature surpasses a certain critical threshold, the risk of health problems, such as disability from back pain and cardiopulmonary compromise, increases. Limited physical function and psychological consequences, such as the poorer perception of body-image, significantly affect patients' quality of life [15], [16].

\footnotetext{
* Corresponding author: Justyna Opydo-Szymaczek, Department of Pediatric Dentistry, Poznan University of Medical Sciences, ul. Bukowska 70,60-812 Poznań, Poland. Phone: +48 61 8547053, fax: +48 61 8547059, e-mail: jopydo@ump.edu.pl

Received: July 23rd, 2019

Accepted for publication: September 27th, 2019
} 
Our understanding of IS etiology is still limited with different theories looking to biomechanical, neuromuscular, genetic, hormonal, and environmental origins [1], [8]. One of the speculations is that IS is caused by environmental stress causing developmental instability and a loss of symmetry in growth [9].

In general, there are three main types of bilateral asymmetry in biology: fluctuating asymmetry (FA), directional asymmetry (DA) and antisymmetry (AS). DA occurs when one structure preferentially outgrows the homologous structure on the contralateral side. IS itself can be an example of a directional asymmetry of the spine since it is classically convex to the right in the thoracic and left in the lumbar region. FA is a model of variation in which deviations from symmetry are distributed close to a mean of zero, and are random and non-directional. FA is assumed to result from the inability of an organism to grow identical bilateral homologous structures, and thus might be used as a marker of the developmental instability. Finally, AS is found whenever one side is usually larger than the other, yet the position of both of them varies randomly in a population, leading to a platykurtic or even bimodal distribution of the differences between the left and right sides of the body [18].

Both fluctuating asymmetry and the presence of increased directional asymmetry were observed in patients affected by IS [9]. Many researchers have also suggested a relationship between a postural deformity of the trunk, such as IS and asymmetric orthodontic conditions [4], [17], [20], [22]. According to a systematic review published in 2011 [21], there is plausible evidence for an increased prevalence of unilateral Angle Class II malocclusions, lateral crossbite, midline deviation as well as reduced range of lateral movements in patients affected by scoliosis. These associations do not necessarily point to causality since it is difficult to determine whether they are primary (playing a role in IS development) or secondary (develop as a result of IS).

The causal relationships between IS and asymmetric features of occlusion have been attributed to compensatory head posture resulting from the pathological curvatures along the spine [2], as well as the influence of craniomandibular abnormalities on the spine development [6].

Unilateral crossbites were interpreted, for example, as a transmission of the asymmetry of the body [11]. However, these relationships in patients with IS are not clear. In the study by Nakashima et al. [17], a positive correlation between the Cobb angle and the degree of mandibular deviation was detected, while Ben-Bassat et al. [4] found no association between location, side, or severity of scoliosis and the appear- ance or side of the malocclusion. Regarding the association between craniofacial morphology and trunk asymmetry, Zepa et al. [25] found that all facial morphologic variables were almost similar in patients with left-sided and right-sided scoliosis.

It is noteworthy that the studies conducted so far did not analyze the occlusion in IS patients within the context of the type of asymmetry. Thus, the purpose of the present study was to quantify left-right dentoalveolar asymmetries in young female patients with IS and to assess the relationship between these asymmetries and the side, location, and severity of scoliosis.

\section{Materials and methods}

The study was approved by the Bioethics Committee at the Poznan University of Medical Sciences (No. 1058/17) and written informed consent was obtained from the parents and 16-year-old patients.

\section{Characteristics of the study group and healthy controls}

The study group was comprised of 60 female patients aged $12-16$ years (mean \pm SD $14.0 \pm 1.3$ ), who visited the pediatric orthopedic outpatient office (Poznań, Wielkopolska Province) for the conservative treatment of IS with a Cheneau brace and had no previous orthodontic treatment. Anterior/posterior spine $\mathrm{X}$-rays were used to assess curve direction, and the severity of IS expressed by the Cobb angle and apical vertebra translation measured, according to the methodology by Harms Study Group [5].

The inclusion criteria were as follows: female gender, age $12-16$ years old, a Cobb angle of $20-40^{\circ}$. Only patients with fully erupted permanent dentition (excluding wisdom teeth) without untreated caries and extensive restorations were included in the study.

Twenty-four patients had thoracic scoliosis, 23 patients had thoracolumbar scoliosis, and the remaining 13 patients had lumbar scoliosis (Th7 was the apical vertebra in 1 patient, Th 8 - in 8 patients, Th 9 - in 10 patients, Th10 - in 4 patients, Th11 - in 1 patient, Th12 - in 9 patients, L1 - in 14 patients, L2 - in 11 patients, and L3 in 2 patients). In 30 patients, scoliosis was convex to the right and in 30 patients to the left. Despite the relatively high representation of levoscoliosis, none of the subjects was diagnosed with a neurological condition or any other health-compromising problem.

The control group of orthopedically 54 healthy females aged 12-16 (mean $14.3 \pm 1.6$ ) with no history of previous orthodontic treatment was recruited in two 
randomly selected schools in Wielkopolska Province. Their selection was carried out by an orthopedic surgeon and a dentist based on the Adam's forward bend test, the measurement of trunk rotation with the use of Bunnel scoliometer and the dental examination. Cases with a trunk rotation exceeding $4^{\circ}$ were excluded from the study [5].

\section{Clinical examination and measurements of asymmetries in teeth relationships}

Apart from clinical examination, alginate impressions and wax bites were taken to measure the left-right asymmetries in teeth relationships. Impressions were poured directly after patient's examination. Measurements were made with the use of digital caliper on fullmouth dental casts by the same dentist "blinded" with regard to whether or not the patients had IS.

The actual dimensions were read directly from the millimeter scale between the short arms of the caliper. Interarch relationships were assessed on the casts in maximum intercuspation. Eight sorts of variables were measured from each subject [10]:

1. Deviation of the incisor midlines (a mandibular shift to the right was given a positive sign) (Fig. 1).

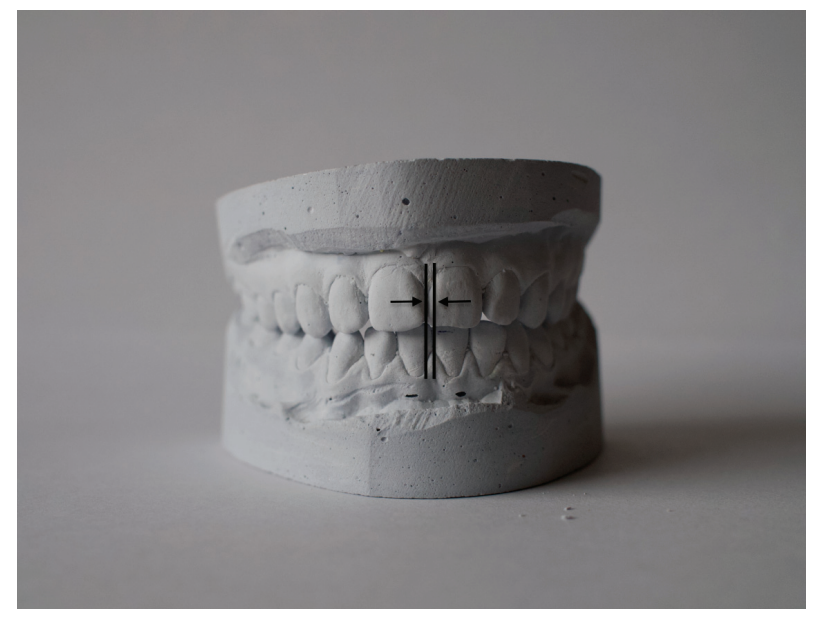

Fig. 1. Illustration of the method of measuring the incisor midline discrepancy

(mandibular shifted to the right was scored as positive)

2. Incisor overjet measured separately on the right and left central incisors (an anterior crossbite was given a negative score) (Fig. 2).

3. The canine deviation which was the horizontal distance from the cusp tip of the upper canine to its normal position in the embrasure between the lower canine and first premolar (the mesial position of the maxillary canine was given a negative score) (Fig. 2).

Buccal segment relation which parallels Angle's molar classification, but on a continuous scale, where the horizontal distance of the buccal groove of the lower first molar is measured relative to the mesiobuccal cusp tip of the upper first molar (Fig. 2). An idealized Class I relationship has a buccal segment relation of $0 \mathrm{~mm}$; Class II relationship was given a negative value.

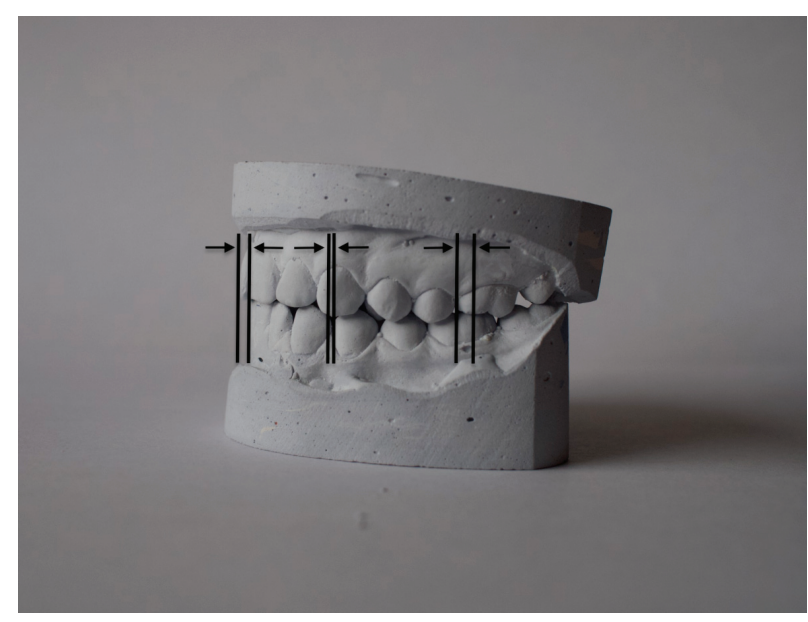

Fig. 2. Illustration of the method of measuring canine deviation (mesial position of the upper canine was given a negative score), overjet (an anterior crossbite was given a negative score), and buccal segment relationship (the lower molar malpositioned to the distal, was given a negative score)

5. Arch chords which were the straight-line distances from the incisive interdental papilla to the distalmost aspect of the canine and the distobuccal aspect of the first molar. Chords were measured in each of the two quadrants of the maxilla and the mandible (Fig. 3).

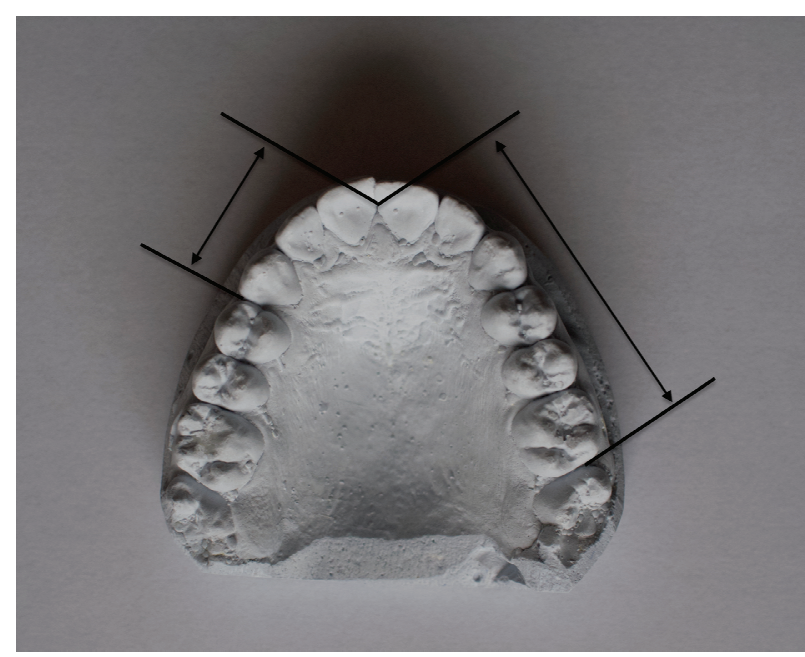

Fig. 3. Illustration of the method of measuring 1-6 and 1-3 arch chords: with one beak of the caliper on the labial interincisal papilla, the other positioned at the distal-most aspect of the canine (the 1-3 chord) and, independently, at the distal-buccal heel of the first molar (the 1-6 chord). Arch chords measurements were made separately on the maxillary and mandibular dental casts 


\section{The technical error of measurement}

The error of measurement was assessed using the conventional Dahlberg statistics [13], namely

$$
D=\sqrt{\sum_{i=1}^{n} \frac{d i^{2}}{2 n}},
$$

where $d i$ is the difference between the first and second measurement of specimen $i ; n$ is the sample size, which was re-measured. The unit of measurement does not cancel out, so $D$ is expressed as an average millimetric difference attributable to measurement imprecision. Double determinations of 114 measurements yielded an average error of just $0.087 \mathrm{~mm}$ (SD $0.046 \mathrm{~mm}$ ). There is no definite reference to decide if some amount of error can be considered acceptable or not. Baumrind and Frantz [3] suggested that differences of measures derived from a patient should be at least the double of the standard deviation of the error of measurement. In this situation, they can be considered as significant. Thus, any significant differences detected during statistical analysis were compared to the double standard deviation of the measurement error $(0.092 \mathrm{~mm})$.

\section{Types of left-right asymmetry}

Three sorts of left-right asymmetry were assessed, namely FA for individual traits and for all traits, DA and AS. FA and AS occur for homologous dimensions when the sample distribution of the left-right differences is centered on zero. DA is identified when the mean or the group median differs significantly from zero based on one sample $t$-test (for data with normal distribution) or a non-parametric equivalent of one sample $t$-test, namely Wilcoxon test for paired-data (for data with abnormal distribution). Preserving the signs of the left-minus-right $(\mathrm{L}-\mathrm{R})$ differences,

$$
\mathrm{DA}=\text { mean }(\mathrm{L}-\mathrm{R}) \text {, }
$$

the magnitude of FA for every dimension was expressed as the absolute value of the side difference of a variable within each case (FA1 index by Palmer) [18]:

$$
\mathrm{FA} 1=\text { mean }|\mathrm{L}-\mathrm{R}| \text {, }
$$

while overall asymmetry in an individual for all traits (apart from traits presenting significant DA) was calculated as a sum of all measured absolute values of the side differences of an individual (FA11 index by Palmer) [18]:

$$
\mathrm{FA} 11=\frac{\Sigma|\mathrm{L}-\mathrm{R}|}{n},
$$

where $n$ was the number of cases in the study.

\section{Statistical analysis}

The data was analyzed statistically by Statistica (version 12) for Windows 10 Home (version 10.0.15063) with significance taken as $p<0.05$.

After performing Levene's test for equality of variances, a one way parametric ANOVA or a nonparametric Kruskal-Wallis ANOVA were used to assess any differences in the mean measurements and magnitudes of asymmetry among various subgroups of IS and the control group. If the statistical difference was detected, post-hoc procedures (Tukey's RIR test or multiple comparisons of mean ranks) were used to compare individual pairs of means.

After performing Shapiro-Wilk normality test, parametric Student's $t$-test for independent variables or non-parametric Mann-Whitney $U$-test were used for evaluation of differences between the control and the study group, while Wilcoxon test for paired data and Student's $t$-test for dependent variables were used to assess differences between right and left parameters in every individual.

Departures from the normality of DA were also evaluated based on kurtosis and visual assessment of the histograms, since platykurtic distribution with negative kurtosis may suggest the presence of AS. AS artificially inflates measures of FA, and therefore any traits exhibiting AS should be excluded from FA analyses [18].

\section{Results}

All detected significant differences exceeded double standard deviation of measurement error $(0.092 \mathrm{~mm})$.

\section{Side specific occlusal dimensions (Table 1)}

Statistically significant differences in the mean midline deviation, buccal segment relation on the left and canine deviation on the left between the control group and the study group were detected ( $p=0.0419$, $p=0.0458, p=0.0204$ ).

An average magnitude of the incisor midline discrepancy (the equivalent of the DA asymmetry of the midline deviation) was positive in subjects from the control group and negative in subjects with IS (tendency to the right and left mandibular shifts, respectively). The buccal segment relation on the left was given an average positive sign (distal shift of the upper molar) in the control group and a negative sign (mesial shift of the upper molar) in a study group. A statistically significant difference between buccal segment 


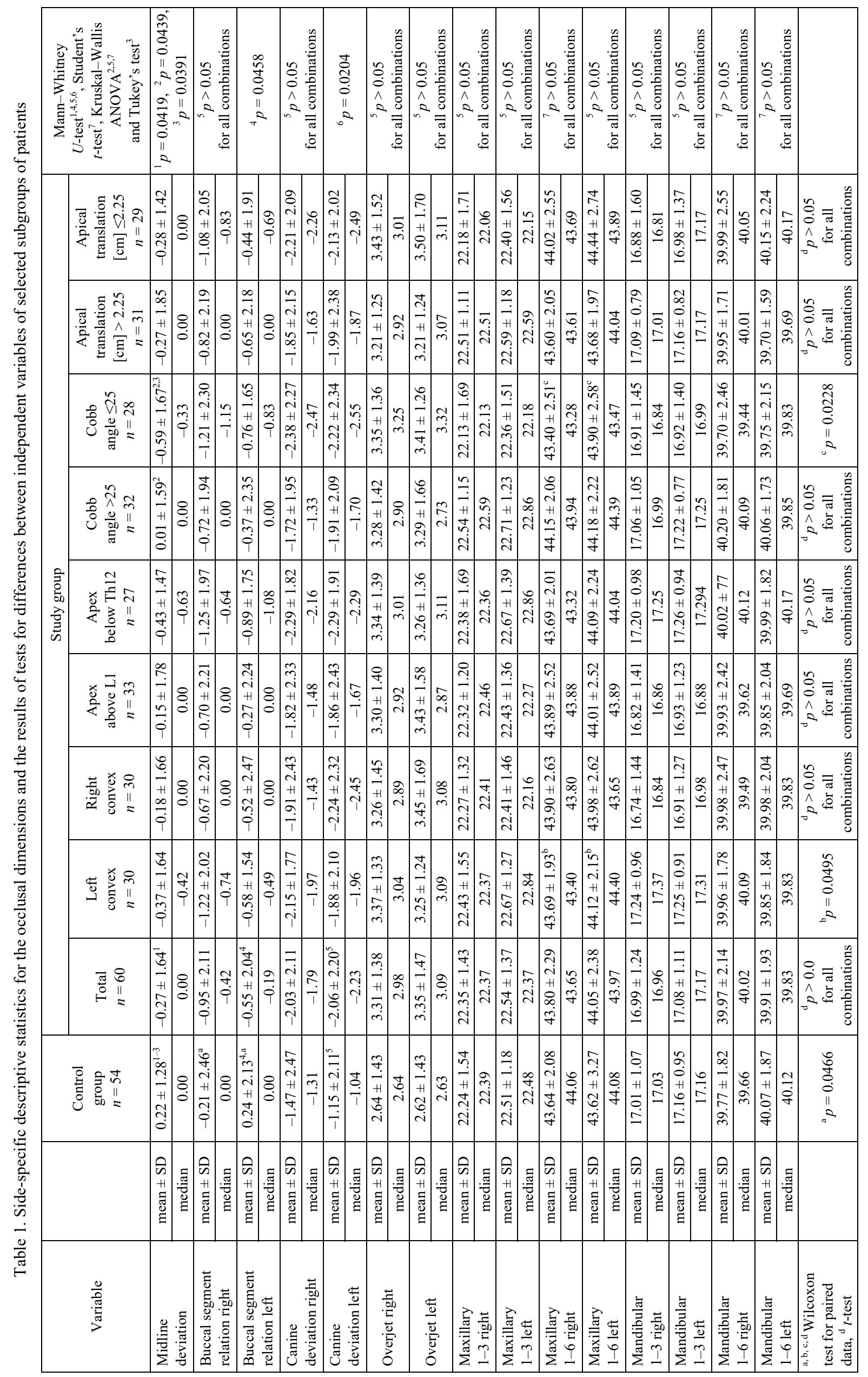




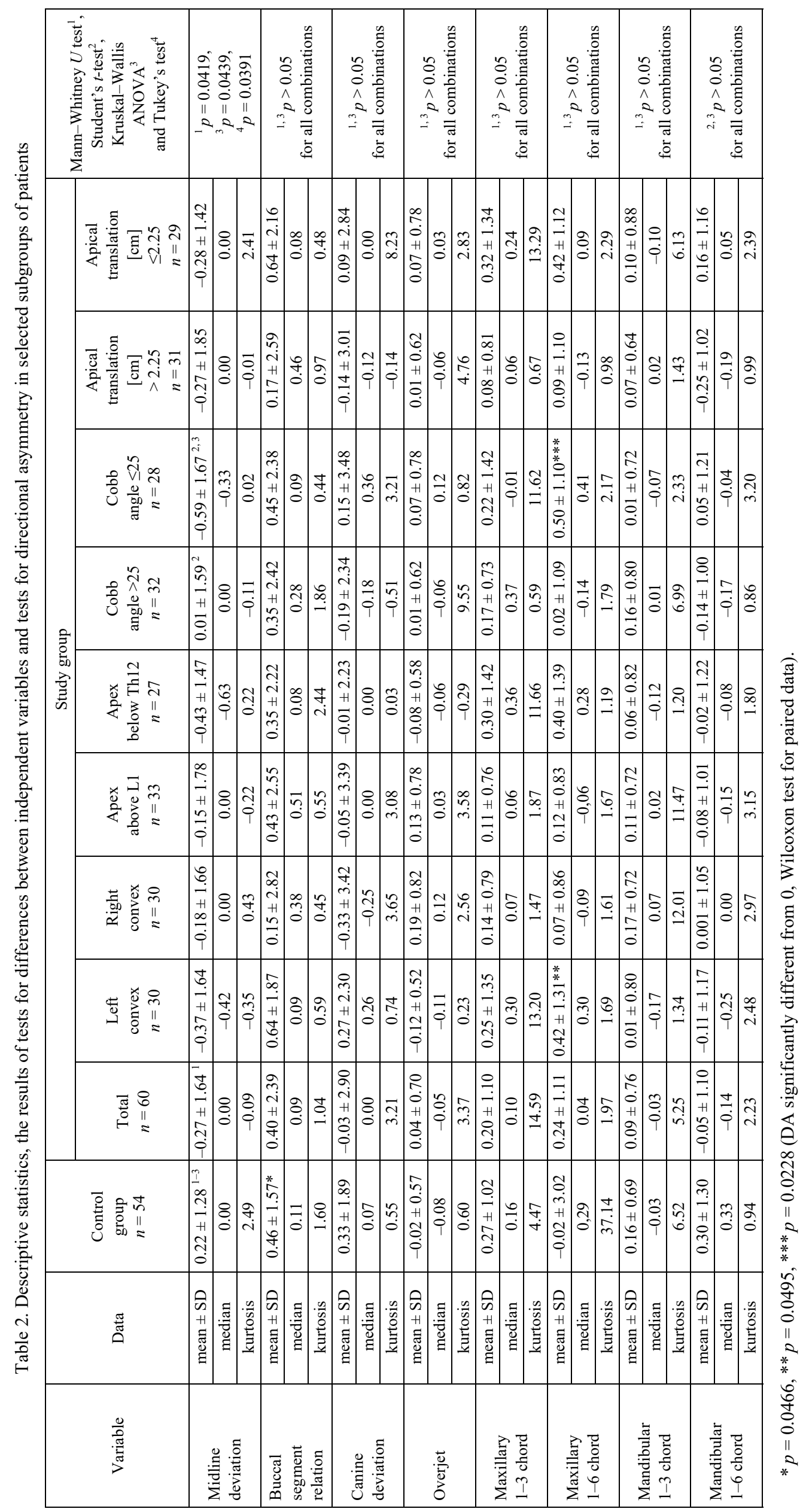




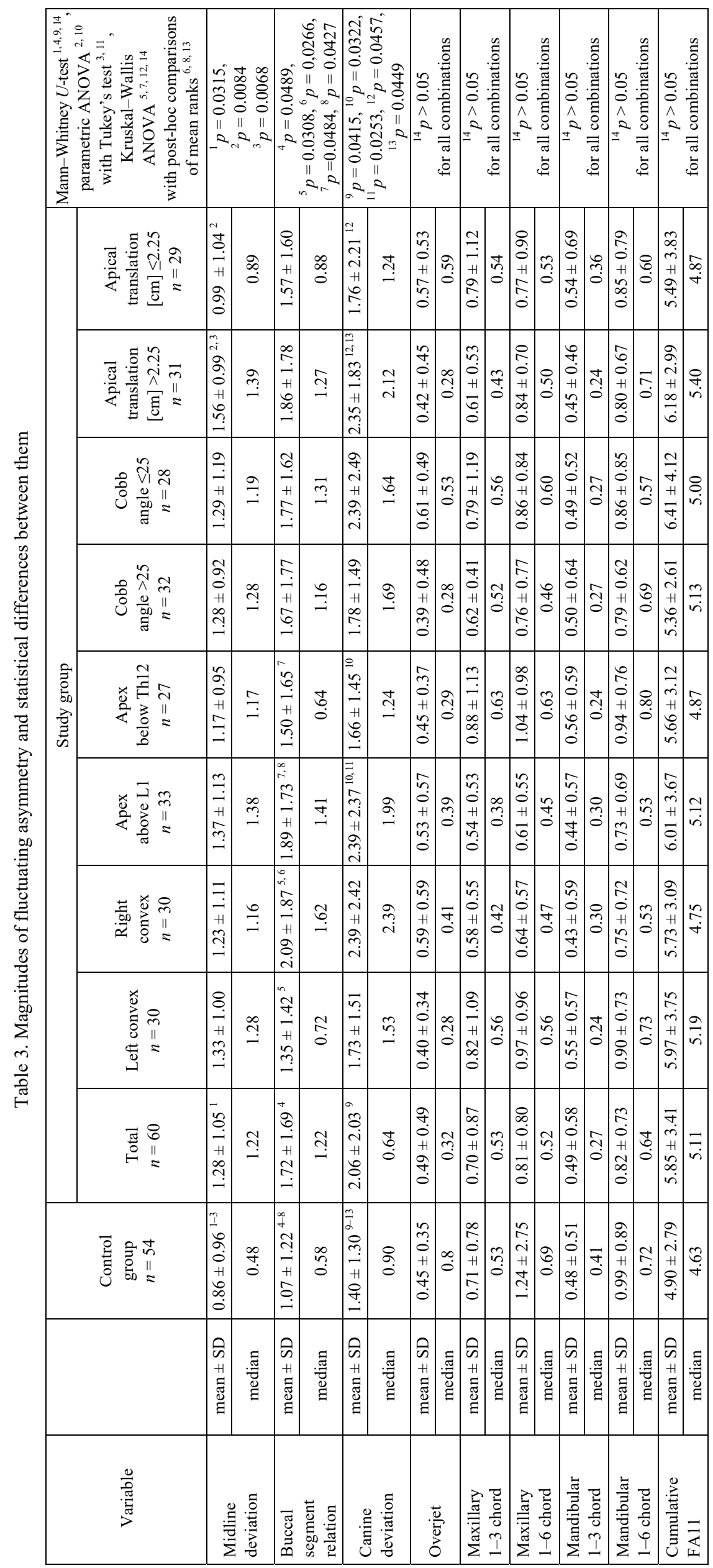


relation on the left and right was detected in the control group of patients $(p=0.0466)$. Left upper canines showed a tendency towards the mesial position in both groups, but were significantly more shifted forward in the study group ( $p=0.0204)$.

Analysis of variance followed by post-hoc multiple comparisons test also revealed a statistically significant difference in the mean midline deviation between the control group and in the group with Cobb angle $\leq 25(p=0.0391)$.

\section{Directional asymmetry (Table 2)}

All of the left-minus-right means of the buccal segment relation were positive showing that the left lower arch was slightly but systematically larger than the right one (all subgroups of patients were systematically more Class II on the right). Left-minus-right canine deviations showed more variable spectrum of values. They were positive in healthy controls and negative in patients with right convex and more severe IS, with no significant differences between groups.

Three measures of asymmetry (buccal segment relation in the control group, a maxillary 1-6 chord in subjects with left convex and subjects with Cobb angle $\leq 25)$ exhibited statistically significant directionality $(p=$ $0.0466, p=0.0495$, and $p=0.0228$, respectively).

Apart from midline deviation (as described earlier), the magnitudes of DA asymmetry did not differ among groups.

\section{Fluctuating asymmetry (Table 3)}

The magnitude of FA1 of the midline deviation and canine deviation were statistically significantly higher for subjects with IS as compared to healthy controls ( $p=0.0315$ and $p=0.0415$, respectively). Subjects with apical translation $>2.5$ showed: higher FA1 of the midline deviation, compared to subjects from the control group $(p=0.0068)$. They also displayed higher FA1 of canine deviation, compared to the control group $(p=0.0449)$. The magnitude of FA1 of buccal segment relation was also significantly higher for subjects with IS, compared to healthy controls $(p=0.0489)$ and for subjects with apex located above L1, compared to the controls $(p=0.0427)$, however, healthy subjects showed DA of buccal segment relation (Table 3), and therefore, this parameter should be excluded from FA analysis.

Cumulative FA11 for all traits (apart from buccal segment relation and maxillary 1-6 chord, which were excluded due to DA) was only slightly non-significantly higher in patients with IS, compared to the control group.

\section{Antisymmetry}

Negative kurtosis suggesting the presence of antisymmetry was detected in 7 DA parameters (Table 2). Graphs of these data were visually inspected, the degree of antisymmetry was minimal, and therefore, all data were included in the analysis of FA1 and FA11.

\section{Discussion}

The results of the study indicate that bilateral asymmetry of occlusion is prevalent in the group of teenage female patients with no previous orthodontic treatment, what was earlier confirmed in other orthodontic studies [10], [12].

Only three measures if DA (buccal segment relation in the control group and maxillary 1-6 chord in subjects with left convex and subjects with Cobb angle $\leq 25$ ) exhibited statistically significant directionality. Harris and Bodford [10] detected directional asymmetry in four of eight measured variables (buccal segment relation, canine deviation, maxillary 1-6 chord, and mandibular 1-6 chord). However, they examined patients with various types of orthodontic problems, among which severe class II malocclusion was associated with significant asymmetries.

Notwithstanding lack of statistically significant DA of most parameters, the observed L - R differences in our control group, where the left lower arch systematically exceeds the size of the right (subjects are significantly more class II on the right), are generally in agreement with those obtained in other orthodontic surveys and seem to be a manifestation of a directional asymmetry [10], [12]. This phenomenon has been attributed to the compensatory adjustments for right hemispheric dominance in the central nervous system [10], [12]. It is noteworthy that subjects with IS presented a different pattern of dentoalveolar asymmetries with mandible shifted to the left and lack of DA of buccal segment relation. Neither direction of the curve nor apical vertebra's location or apical translation significantly affected the magnitude of asymmetries, however, analysis of the data clearly shows that opposite (left) direction of mandibular shift and significant opposite DA of maxillary 1-6 chord was a feature of subjects with lumbar scoliosis, less severe (Cobb angle $\leq 25$ ), convex to the left. Whether it could be an example of transmission of the asymmetry of the body, remains a matter of debate.

Orthopedic studies also revealed a tendency towards specific directional bilateral asymmetries in general population. According to Doi et al. [7], IS can 
be a manifestation of increased innate DA, since trunk asymmetry with a right thoracic curvature resembling the deformity in IS is widely detected in the normal spine. It seems that the native curvature or rotatory pattern of the spine is an important factor for the direction of spinal decompensation. Goldberg et al. [18] suggested that, in scoliosis, children with an increased left-right developmental gradient loss, when stressed by the adolescent growth spurt, symmetry of the spine down that gradient. Individuals with higher developmental stability will need greater stress for a major symmetry loss. One could hypothesize that the development of dental arches of the IS patients might be disturbed by the same stressing factors, which will result in an increased frequency of dentoalveolar asymmetries. Of all three types of bilateral asymmetry, only FA has been considered as a proper biomonitoring tool to measure developmental instability of an individual, i.e., the inability of an organism to cope with perturbation during development. DA and AS may have a significant genetic basis and, therefore, are harder to associate with developmental instability [18].

Regarding FA, the mean absolute values of the midline deviation, canine deviation, and buccal segment relation were statistically significantly higher for subjects with IS, compared to the healthy controls (although the last parameter should be excluded from the analysis of the control group as an example of DA asymmetry). Specifically, patients with more severe scoliosis (apical translation $>2.5 \mathrm{~cm}$ ) had higher FA of the midline shift and canine deviation, while the thoracic apex of curvature was related to the higher FA of the buccal segment relation. Thus, IS patients exhibited lower developmental stability of some parameters, although their cumulative FA for all traits was only slightly non-significantly higher compared to the control group.

It should be noticed that FA, which is thought to result from the accumulation of minor stochastic events during development, turned out to be the dominant type of asymmetry detected in both groups of patients. As suggested by Harris and Bodford [10], there is a number of environmental issues that may cause FA, such as side differences in times of primary tooth exfoliation (or extraction), position and orientation of the developing successor's tooth buds, differences in eruptive tempos and pathways, differences in tooth emergence and sequence, positions of antagonists. Occlusion and teeth of patients with IS are additionally subjected to abnormal forces resulting from a body and head tilt to one side and forward head posture. As suggested by Solow and Sonnensen [23], an extension of the craniocervical posture leads to passive stretching of the skin, muscles, and fascia, that cover the head and neck. Stretching of this convex soft tissue layer creates a dorsally directed force which impedes the forward-directed growth of the facial skeleton and, in particular of the mandible. Consequently, patients with IS have been reported to present distal molar occlusion (Angle Class II), more frequently [21], [22]. Our results are in agreement with these observations. The magnitude of upper molars and canines mesial position in individuals with IS was higher, compared to the controls. Consequently, higher FA of some dentoalveolar parameters observed in IS patients might be a result of their tendency to Angle Class II malocclusion, where anterior teeth have no functioning antagonists for guidance and stability [10].

According to a recently published systematic review, there is only low-quality evidence suggesting a higher prevalence of occlusal alterations among patients with spinal deformity than in controls. Methodological limitations of the studies reviewed included small sample size, lack of the control group, absence of statistical data, incomplete description of the study sample, lack of radiological confirmation of the spinal deformity, no information on the presence and type of the bracing [14]. The last can be a significant confounding factor since the use of the original Milwaukee brace in scoliosis therapy has been demonstrated to negatively affect occlusion [19].

Our study also has several limitations that should be considered in the interpretation of the results. We investigated a relatively small number of female patients with low-grade scoliosis, which may limit the generalizability of the findings. On the other hand, our inclusion criteria eliminated possible confounding factors related to changes in dental relationships and occlusion after primary teeth exfoliation, teeth migration due to large cavities, effects of different type of orthopedic treatment as well as gender-related differences in bones growth.

\section{Conclusions}

Our results revealed that dentoalveolar asymmetries are commonly encountered in young female patients, regardless of the presence of IS. This emphasizes the need for orthodontic screening in all teenage patients. Individuals with IS seem to present with higher tendency to Angle Class II malocclusion, with a different pattern of DA, as well as higher FA of incisor midline discrepancy and canine deviation parameters as compared to the controls. 


\section{Acknowledgements}

The authors would like to thank the patients who participated in the study, the staff of the schools and the staff of the Department of Pediatric Dentistry who assisted in the project.

\section{References}

[1] Amat P., Occlusion and posture: facts and beliefs, J. Dentofacial Anom. Orthod., 2008, 11, 186-211.

[2] Arienti C., Villafańe J.H., Donzelli S., Zaina F., BURASCHI R., NEGRINI S., Trunk and craniofacial asymmetry are not associated in the general population: a cross-sectional study of 1029 adolescents, Eur. J. Med. Res., 2017, 22 (36), DOI: 10.1186/s40001-017-0280-y.

[3] BAUMrind S., Frantz R.C., The reliability of head film measurements. Conventional angular and linear measurements, Am. J. Orthod., 1971, 60, 505-517.

[4] Ben-Bassat Y., Yitschaky M., KaPlan L., BRin I., Occlusal patterns in patients with idiopathic scoliosis, Am. J. Orthod. Dentofac. Orthop., 2006, 130, 629-633.

[5] D'Andrea L.P., Betz R.R., Lenke L.G., Clements D.H, Lowe T.G., Merola A., Haher T., Harms J., Huss G.K., Blanke K., MCGlothlen S., Do radiographic parameters correlate with clinical outcomes in adolescent idiopathic scoliosis?, Spine, 2000, 25, 1795-1802.

[6] D’Attilio M., Filippi M.R., Femminella B., Festa F., TECCO S., The influence of an experimentally-induced malocclusion on vertebral alignment in rats: a controlled pilot study, Cranio, 2005, 23, 119-129.

[7] Doi T., Harimaya K., Mitsuyasu H., Matsumoto Y., Masuda K., KobayaKawa K., Iwamoto Y., Right thoracic curvature in the normal spine, J. Orthop. Surg. Res., 2011, 6 (4), DOI: 10.1186/1749-799X-6-4.

[8] Fadzan M., Bettany-Saltikov J., Etiological Theories of Adolescent Idiopathic Scoliosis: Past and Present, Open Orthop. J., 2017, 11, 1466-1489.

[9] Goldberg C.J., Dowling F.E., Fogarty E.E., Moore D.R., Adolescent idiopathic scoliosis as developmental instability, Genetica, 1995, 96, 247-255.

[10] HARris E.F., BODFORD K., Bilateral Asymmetry in the Tooth Relationships of Orthodontic Patients, Angle Orthodontist, 2007, 77, 779-786, DOI: 10.2319/081606-335.

[11] HiRSCHFELDER U., HiRSCHFELDER H., Effects of scoliosis on the facial bones, Fortschr Kieferorthop, 1983, 44, 457-467.

[12] Hujoel P.P., Masterson E.E., Bollen A.M., Lower face asymmetry as a marker for developmental instability, Am. J. Hum. Biol., 2017, 29, DOI: 10.1002/ajhb.23005.
[13] Kiм H.Y., Statistical notes for clinical researchers: Evaluation of measurement error 2: Dahlberg's error, BlandAltman method, and Kappa coefficient, Restor. Dent. Endod., 2013, 38, 182-185, DOI: 10.5395/rde.2013.38.3.182.

[14] Langella F., Fusini F., Rossi G., VillafańE J.H., Migliaccio N., Donzelli S., Berjano P., Spinal deformity and malocclusion association is not supported by high-quality studies: results from a systematic review of the literature, Eur. Spine J., 2019, DOI, 10.1007/s00586-019-05896-4.

[15] Misterska E., GŁowacki J., GŁowacki M., Okręt A., Long-term effects of conservative treatment of Milwaukee brace on body image and mental health of patients with idiopathic scoliosis, PLOS One, 2018, 13(2), DOI: 10.1371/ journal.pone.0193447.

[16] Misterska E., GŁowacki J., OKręt A., LaurentowsKa M., GŁOWACKI M., Back and neck pain and function in females with adolescent idiopathic scoliosis: A follow-up at least 23 years after conservative treatment with a Milwaukee brace, PLOS One, 2017, 12(12), DOI: 10.1371/journal.pone.0189358.

[17] NAKashima A., NaKano H., Yamada T., InOUE K., SugiYama G., Kumamaru W., Nakajima Y., Sumida T., Yokoyama T., Mishiama K., Mori Y., The relationship between lateral displacement of the mandible and scoliosis, Oral Maxillofac. Surg., 2017, 21, 59-63, https://www.ncbi.nlm.nih. gov/pubmed/28039546.

[18] Palmer R., Strobeck C., Fluctuating Asymmetry Analyses Revisited, [in:] M. Polak (Ed.), Developmental Instability (DI): Causes and Consequences, Oxford University Press, 2004.

[19] PAVsic J., The effect of orthodontic measures on the growth of the mandible in scoliosis patients during treatment with the Milwaukee brace, Stomatol. DDR, 1983, 33, 676-682.

[20] Pećina M., Lulić-Dukić O., PećINA-Hrncević A., Hereditary orthodontic anomalies and idiopathic scoliosis, Int. Orthop., $1991,15,57-59$.

[21] Saccucci M., Tettamanti L., Mummolo S., Polimeni A., Festa F., Tecco S., Scoliosis and dental occlusion: a review of the literature, Scoliosis, 2011, 6 (15), DOI: 10.1186/ 1748-7161-6-15.

[22] Segatto E., Lippold C., VéGH A., Craniofacial features of children with spinal deformities, BMC Musculoskelet. Disord., 2008, 22 (9), DOI: 10.1186/1471-2474-9-169.

[23] Solow B., Sonnesen L., Head posture and malocclusions, Eur. J. Orthod., 1998, 20, 685-693.

[24] WiLCZyŃSKi J., BIENIEK K., Correlations of somatic traits and postural defects in girls and boys aged 10-12, Acta. Bioeng. Biomech., 2019, 21 (1), 79-86.

[25] Zepa I., Hurmerinta K., Kovero O., Nissinen M., KönÖnen M., Huggare J., Trunk asymmetry and facial symmetry in young adults, Acta Odontol. Scand., 2003, 61, 149-153. 